\title{
Natural History of Endoscopic Third Ventriculostomy in Adults: Serial Evaluation with High-Resolution CISS
}

\author{
(D) M. Trelles, (D)A.K. Ahmed, (D) C.H. Mitchell, (DI. Josue-Torres, (DD. Rigamonti, and (D) A.M. Blitz
}

\begin{abstract}
BACKGROUND AND PURPOSE: Endoscopic third ventriculostomy is a well-accepted treatment choice for hydrocephalus and is used most frequently with a known impediment to CSF flow between the third ventricle and basal cisterns. However, there are scarce data on the imaging evolution of the defect in the floor of the third ventricle and how this affects patency rates and clinical outcomes. The purpose of this study was to assess whether, and how, the endoscopic third ventriculostomy defect changes in size with time.
\end{abstract}

MATERIALS AND METHODS: All high-resolution endoscopic third ventriculostomy protocol MRIs performed between 2009 through 2014 were retrospectively identified. Two fellowship-trained neuroradiologists, blinded to clinical information, independently reviewed all retrospective cases.

RESULTS: A total of 98 imaging studies were included from 34 patients. The average change in the area throughout the studied period was $0.02 \mathrm{~mm}^{2} /$ day $\left(7.5 \mathrm{~mm}^{2} /\right.$ year), with a higher increase in size noted in the first 3 postsurgical months, with a gradual decrease in the degree of defect-size change. Use of the NICO Myriad device was correlated with the area of the endoscopic third ventriculostomy defect on the last follow-up, demonstrating a larger final defect size in patients in whom the surgical technique included debridement of the endoscopic third ventriculostomy defect walls with the NICO Myriad device ( 28.21 versus $11.25 \mathrm{~mm}, P<.05$ ).

CONCLUSIONS: High-resolution MR imaging with sagittal CISS images is useful in the postoperative evaluation of endoscopic third ventriculostomies. Such findings may prove useful in determining the optimal duration of follow-up with MR imaging of patients who have undergone endoscopic third ventriculostomy.

ABBREVIATION: ETV = endoscopic third ventriculostomy

E ndoscopic third ventriculostomy (ETV) is a commonly performed procedure for the treatment of noncommunicating adult hydrocephalus when the obstruction is between the third ventricle and the basal cisterns. The procedure avoids insertion of a foreign body with its inherent shunt-related complications, such as obstruction, disconnection, catheter migration, infection, and over- or underdrainage. ${ }^{1-3}$ ETV is a well-accepted treatment choice for hydrocephalus and is used most frequently in the presence of a known impediment to CSF flow between the third ventricle and the basal cisterns. ${ }^{4-8}$

Management of patients who have undergone ETV is not sim-

Received April 20, 2018; accepted after revision August 17.

From the Departments of Radiology and Radiological Sciences (M.T., A.M.B.), Neurosurgery (A.K.A., I.J.-T., D.R.), and Neurology (C.H.M.), Johns Hopkins University School of Medicine, Baltimore, Maryland.

Please address correspondence to Ari M. Blitz, MD, Department of Radiology and Radiological Science, Johns Hopkins University School of Medicine, $733 \mathrm{~N}$ Broadway, Baltimore, MD 21205; e-mail: Ablitz1@jhmi.edu

http://dx.doi.org/10.3174/ajnr.A5861 ple because symptoms may be attributable to failure of the ETV, recurrent symptoms from an intracranial process not treated or not fully treated by ETV, or another etiology entirely. Spontaneous closure of a once-patent ETV has also been reported, sometimes with fatal consequences. ${ }^{9,10} \mathrm{MR}$ imaging may provide invaluable information in the assessment of patients with ETV because patency of the ETV defect can be assessed noninvasively. Postoperative imaging has mainly been directed at detecting a CSF flow void in the floor of the third ventricle. At our institution, due to the frequency of the request, a clinical "high-resolution endoscopic third ventriculostomy" protocol MR imaging (ETV protocol) has been established to aid in the evaluation of the patency of the ETV defect. The ETV protocol includes both high-spatialresolution isotropic CISS imaging through the sagittal midline structures and cardiac-gated phase-contrast imaging through the floor of the third ventricle in the expected region of the ETV defect in addition to standard components of head MR imaging. High-resolution CISS permits identification of the anatomic patency of the surgical 
defect as well as its size, and phase-contrast imaging provides complementary data with respect to CSF movement through the ETV defect.

There are scarce data on the imaging evolution of the defect in the floor of the third ventricle and how this affects patency rates and clinical outcomes. The purpose of this study was to assess whether, and how, the ETV defect changes in size with time.

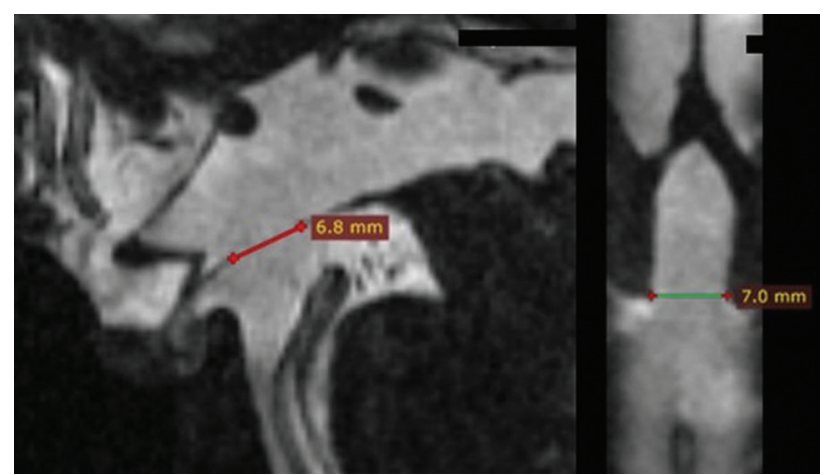

FIG 1. Representative sagittal CISS image and coronal MPR reconstruction of a third ventriculostomy defect with anteroposterior and third ventriculostomy defect size measurement.

Table 1: Demographic information

\begin{tabular}{lccc}
\hline \multicolumn{1}{c}{ Demographics } & $\%$ & No. \\
\hline Sex & $44 \%$ & & 15 \\
$\quad$ Male & $56 \%$ & & 19 \\
$\quad$ Female & & $54(19-76)$ & \\
Mean age (range) (yr) & & \\
\hline
\end{tabular}

Table 2: Site of obstruction to the flow of CSF

\begin{tabular}{lrr}
\hline Location of Obstruction & $\%$ & No. \\
\hline Cerebral aqueduct & $82 \%$ & 28 \\
Fourth ventricle outflow & $3 \%$ & 1 \\
Prepontine cistern & $9 \%$ & 3 \\
Unknown & $3 \%$ & 1 \\
Other & $3 \%$ & 1 \\
& & 34 \\
\hline
\end{tabular}

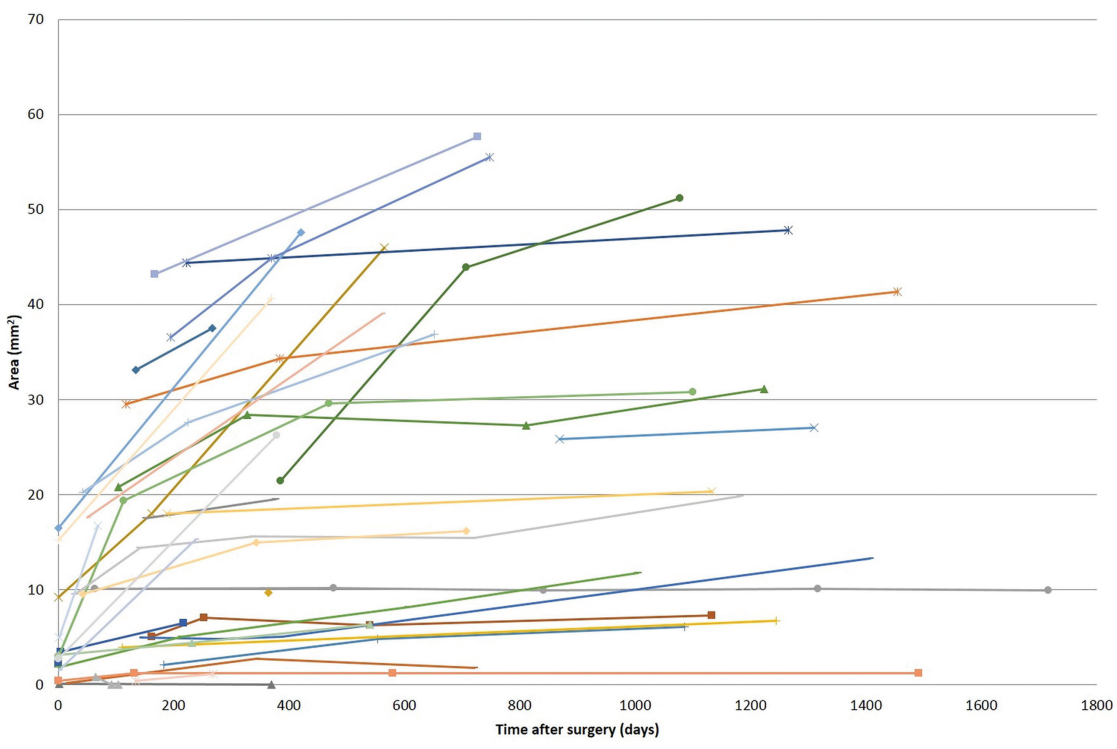

FIG 2. Defect size area across time after surgery.

\section{MATERIALS AND METHODS \\ Patient Selection}

All high-resolution endoscopic third ventriculostomy protocol MRIs performed between 2009 through 2014 at a single institution were identified following institutional review board approval. Two fellowship-trained neuroradiologists, blinded to clinical information, independently reviewed all retrospective cases. Patients with at least 2 postoperative ETV protocol studies were included. Patients who had previously undergone an intracranial operation before ETV, including ventriculostomy tube placement, were excluded. Cases with imaging performed on scanners other than 3T (ie, 1.5T) were excluded. Studies with significant motion (grade 3) were also excluded. The birth date, sex, and date of the operation were obtained from the patient's chart. The site of obstruction to the flow of CSF was obtained from presurgical imaging.

The clinical course defined by the treating physician as improved, stable, or worse was obtained from the chart including: headache, dizziness, nausea, vision deficit, gait problems, urinary incontinence, cognitive decline, other clinically relevant symptoms (ie, irritability, drowsiness, personality changes, seizures), and general clinical outcome. Symptom improvement, stability, or worsening was noted at the last follow-up; thus, patients with initial improvement who, during the follow-up course of the disease, had gradual symptom decline back to baseline status were defined as "stable." During the studied period, the neurosurgeons changed their surgical technique, adopting the NICO Myriad device (NICO Corporation, Indianapolis, Indiana) to debride the wall of the ventriculostomy site; the use of this device was also noted. ${ }^{11}$

\section{Imaging Analysis}

High-resolution isotropic imaging in CISS was performed at 3T (MAGNETOM Trio, Siemens, Erlangen, Germany) field with 0.5 - to 0.6 - $\mathrm{mm}$ near-isotropic image resolution. Imaging parameters consisted of the following: TR/TE, $6.16 / 2.85 \mathrm{~ms} ; 0.5-\mathrm{mm}$ isotropic voxels; flip angle, $40^{\circ}$; FOV, $160 \mathrm{~mm}$; matrix, $320 \times 320$. The presence of patient motion was graded from 1 to 3 ( 1 no motion; 2, mild motion; 3 , severe motion). Using multiplanar reformatting, we identified the presence of a patent ETV defect; if it was present, the greatest anteroposterior (AP) diameter was measured on sagittal images and the greatest transverse (AP) diameter was measured on coronal reformatted images (Fig 1). Measurements were performed by an experienced neuroradiologist (M.T.). To assess the reproducibility of the measurements, a second radiologist (C.H.M.) repeated the measurements blinded to the prior results. The area of the defect was estimated using the ellipsoid formula: $\pi \times \frac{A P}{2} \times \frac{T V}{2}$.

\section{Statistical Analysis}

Categoric variables are presented as percentages; continuous variables are presented as mean $\pm 95 \%$ confidence inter- 


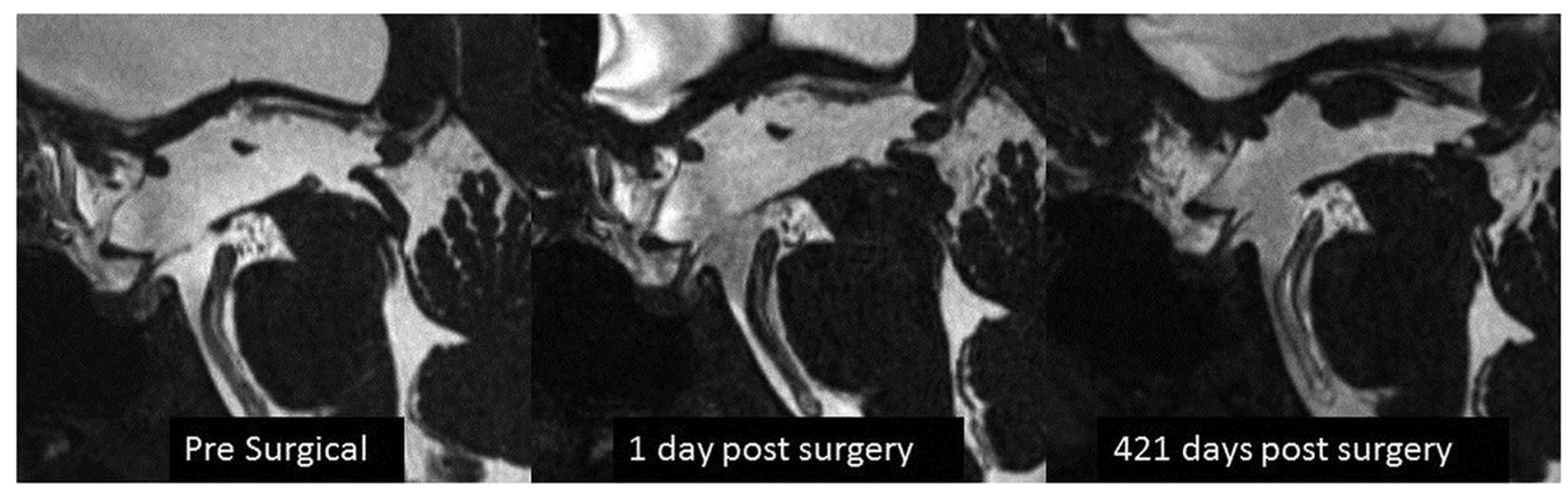

FIG 3. Representative case showing presurgical imaging, first follow-up at 1 day postsurgery, and final follow-up at 421 days postsurgery showing an increase in the ETV defect size.

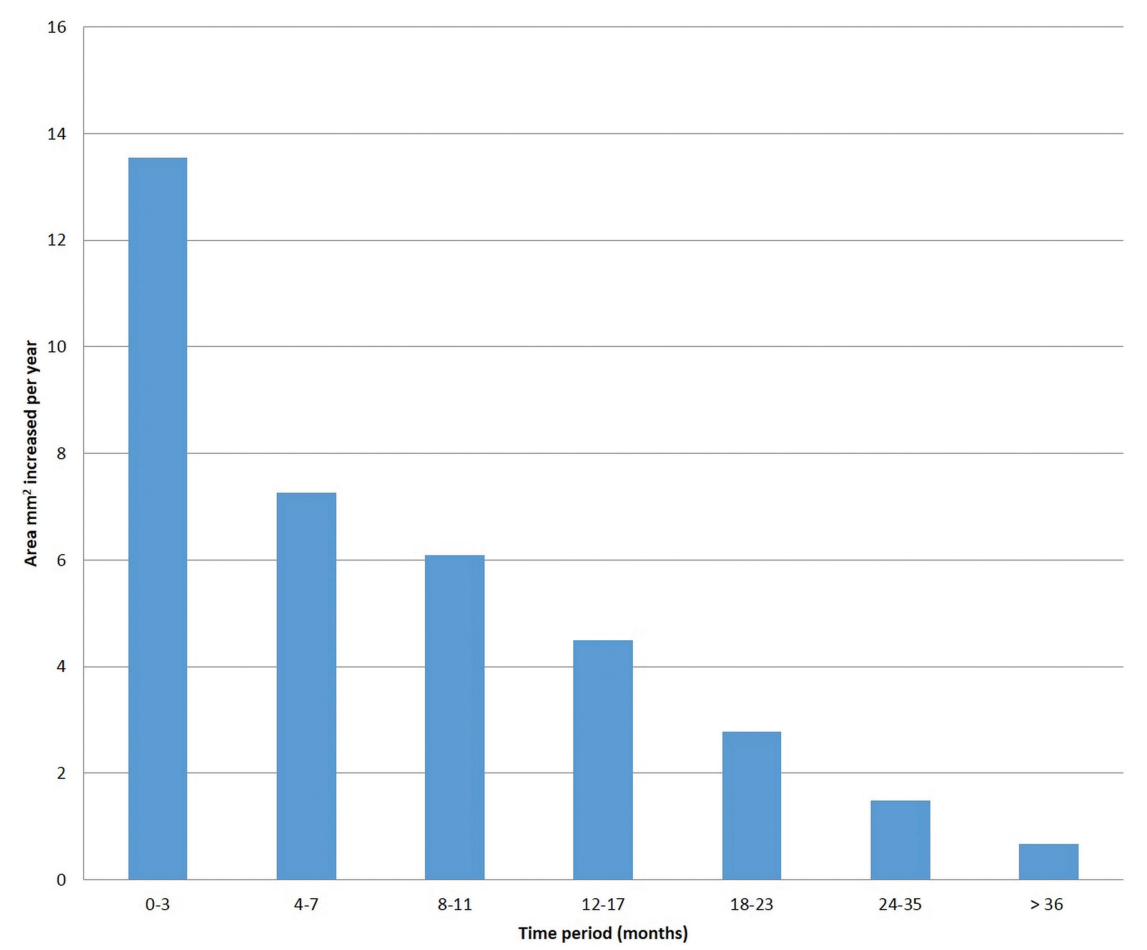

FIG 4. Average increase in the size of the defect across time.

val. The unpaired Student $t$ test with 2 tails was used to test the statistical independence between groups. The Pearson correlation coefficient was used to measure the strength of the association between age and defect size. Interreader agreement was determined by the intraclass correlation coefficient for the area measurement. A value of $P<.05$ was considered statistically significant. Data analysis was performed with SPSS Statistics (Version 20; IBM, Armonk, New York). Graphs were constructed with Excel (Version 14; Microsoft, Redmond, Washington).

\section{RESULTS}

A total of 295 high-resolution ETV protocol MR imaging studies were identified in 84 patients. Fifty patients with 168 studies had prior intracranial operations or $<2$ postoperative studies, and 2 studies had severe motion and were excluded, leaving 125 studies in 34 patients (Table 1). Of these, 27 had been misclassified at the time of protocol code insertion and were preoperative studies. We analyzed the remaining 98 postsurgical ETV protocol MRIs in 34 patients. The mean age was 54 years (range, 19-76 years) with a male/female ratio of 0.78 as shown in Table 1. The overall mean follow-up time, after ETV, was $25.3 \pm 2.8$ months. Follow-up did not significantly differ between those undergoing ETV with or without the NICO Myriad device $(P>.05)$.

The location of obstruction to the flow of CSF is shown in Table 2. Obstruction was located at the cerebral aqueduct in 28 patients (82\%), at the fourth ventricle outflow in 1 patient (3\%), at the prepontine basal cistern in 3 patients (9\%), at another site in 1 patient (3\%), and was unknown in 1 patient (3\%) who had the clinical diagnosis of normal pressure hydrocephalus. Prepontine obstruction occurred due to mechanical obstruction by thickened arachnoid membranes or arachnoidal cysts. $^{5}$

Two patients had their ETVs occlude during the first year of follow-up. All other patients showed an increase in the size of the ETV defect during the imaging time (Figs 2 and 3 ). The average change in the area throughout the studied period was $0.02 \mathrm{~mm}^{2} /$ day $\left(7.5 \mathrm{~mm}^{2} /\right.$ year) with a higher increase in size noted in the first 3 postsurgical months, with a gradual decrease in the degree of defect-size change as seen in Fig 4. Thirteen patients underwent a study in the 2 days following the operation, showing an average defect size of $2.2 \times 2.4 \mathrm{~mm}$ (mean area, $4.6 \mathrm{~mm}^{2}$; range, $0.3-13.0 \mathrm{~mm}^{2}$ ). The intraclass correlation coefficient between both readers in the anteroposterior and third ventriculostomy measurements was 0.83 and 0.88 , respectively.

The age at the operation was correlated to the area of the defect on the last follow-up examination showing a positive correlation, with older individuals having larger defects $(R=0.625$, a moderate positive correlation; $P<.01$; Fig 5 ).

Use of the NICO Myriad device was correlated with the area of 
Max of Area

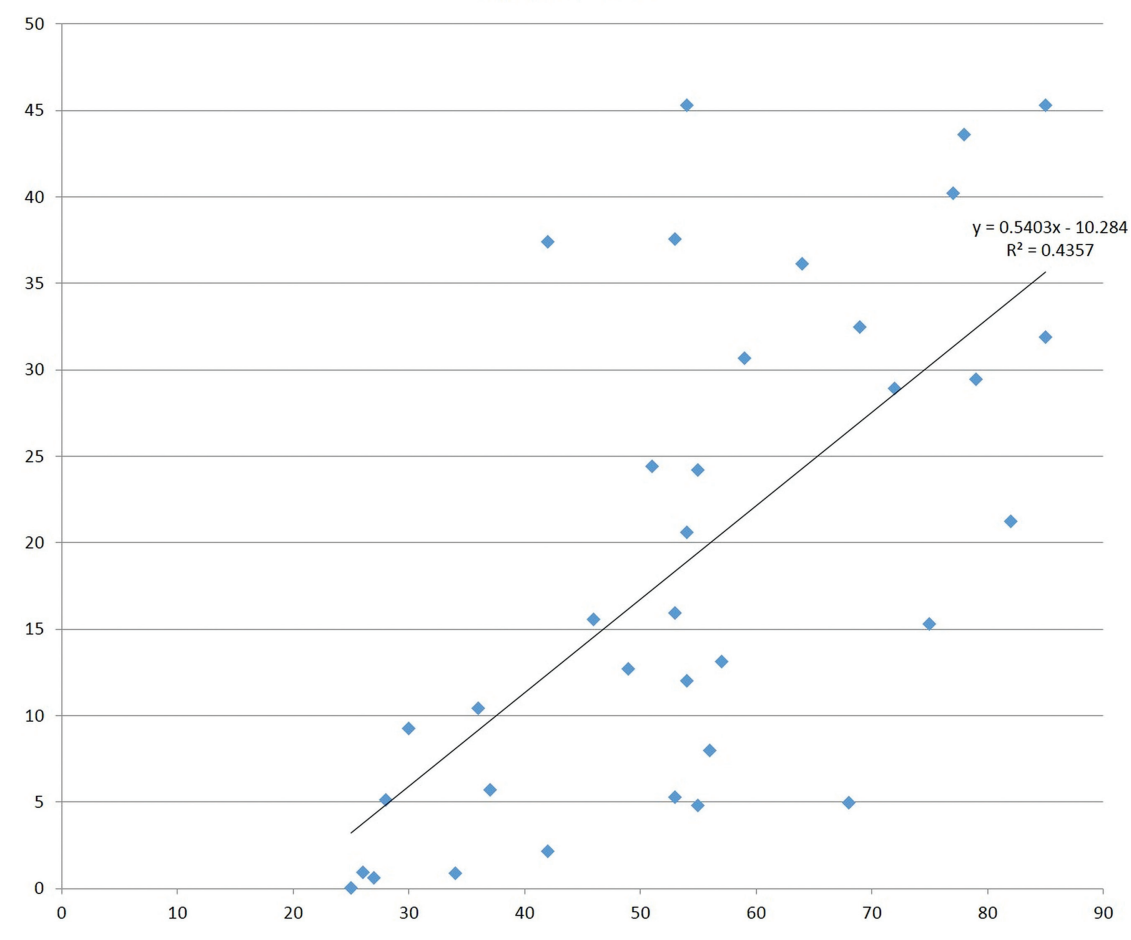

FIG 5. Maximum defect size and age at the operation.

Table 3: Average defect size $\pm 95 \%$ confidence interval and $t$ test for the NICO Myriad device at last follow-up

\begin{tabular}{lccc}
\hline & \multicolumn{3}{c}{ NICO Myriad Device Used } \\
\cline { 2 - 4 } & Yes & No & $\boldsymbol{P}$ Value \\
\hline $\begin{array}{l}\text { Average defect size at } \\
\text { LFU (mean) }\end{array}$ & $28.21 \pm 7.48$ & $11.25 \pm 6.80$ & $<.05$ \\
\hline Note- LFU indicates last follow-up. & & & \\
\hline
\end{tabular}

Note:- LFU indicates last follow-up.

Table 4: Presenting symptoms

\begin{tabular}{|c|c|c|c|}
\hline & $\begin{array}{c}\text { NICO Myriad } \\
\text { Used }\end{array}$ & $\begin{array}{l}\text { No NICO } \\
\text { Myriad }\end{array}$ & $P$ Value \\
\hline Headache & $60 \%$ & $75 \%$ & $>.05$ \\
\hline Dizziness & $26.7 \%$ & $30 \%$ & $>.05$ \\
\hline Nausea & $26.7 \%$ & $35 \%$ & $>.05$ \\
\hline Vision deficit & $40 \%$ & $45 \%$ & $>.05$ \\
\hline Gait abnormality & $53.3 \%$ & $50 \%$ & $>.05$ \\
\hline Urinary incontinence & $46.7 \%$ & $35 \%$ & $>.05$ \\
\hline Cognitive dysfunction & $53.3 \%$ & $60 \%$ & $>.05$ \\
\hline
\end{tabular}

the ETV defect on the last follow-up (Table 3), showing a larger final defect size in patients in whom the surgical technique included debridement of the ETV defect walls with the NICO Myriad device ( 28.21 versus $11.25 \mathrm{~mm}, P<.05$ ).

The most common presenting symptoms were headache, cognitive dysfunction, and gait deficits (Table 4). There were no significant differences in presenting symptoms between patients undergoing ETV with and without the NICO Myriad device $(P>.05$ for all). For all symptoms assessed (ie, headache, dizziness, nausea, vision deficit, gait abnormality, urinary incontinence, cognitive dysfunction), most patients experienced symptom improvement at 6 months following ETV. Most interesting, there was a minor reduction in symptom improvement at last follow-up, which tended to favor the use of the NICO Myriad device (Table 5). However, due largely to sample size, there were no significant differences in symptom improvement between the 2 follow-up time points or with respect to use of the NICO Myriad device $(P>.05$ for all).

\section{DISCUSSION}

This is the first article that, to our knowledge, follows a large group of patients with ETV who underwent repeat MR imaging. With the exception of 2 patients who had occluded ETVs, the remainder showed an increase in size of the ETV defect during the imaging period.

Why the ETV defects enlarged with time is unknown. On surgical videos from ETV procedures after the ETV defect has been created, one can sometimes see significant CSF pulsation causing inward and outward movement of the floor of the third ventricle. ${ }^{2,12-17}$ We postulate that such CSF pulsation and the resultant shear forces, when aggregated across days to months to years, could cause widening of the ETV defect. Our data showed a tendency toward a greater increase in the ETV defect area in older individuals, perhaps reflecting a decreased ability of the ETV margin in older individuals to withstand such small shear stresses. Our data suggest that not all ETVs showed an increase in size. It is uncertain at this time whether the ETV defect size or the velocity of the ETV defect-size change influences disease progression, or if either size or velocity of change are related to clinical outcomes.

The occlusion rate observed in this study was low (2/34 patients or 6\%), partly because some patients with occluded ETVs did not undergo $>1 \mathrm{MR}$ imaging and thus were not included in our studied population. This finding is similar to those in reports in the literature, however, which cite $\sim 9 \%$ occlusion during a median follow-up of 8.5 months and older age as protective against shunt revision. ${ }^{18}$ Nonetheless, no patients had an occlusion 1 year after the operation, potentially reducing the need for imaging follow-up to evaluate obstruction after this time. Both patients with occluded ETVs were the youngest in the group at 19 and 22 years of age. The NICO Myriad device was not used in their operations. No other distinguishing feature was found. Similar to previous reports, use of the NICO Myriad device, an aspirating/ resecting device to debride the walls of the ventriculostomy site, was associated with the area of the defect size on last follow-up. ${ }^{11}$

Although not the main purpose of this study, symptom improvement increased at 6 months following ETV, but improvement diminished slightly at the last follow-up visit. There seemed to be greater sustained improvement with the use of the NICO Myriad device, but this was not statistically significant, due to the small sample size. The main limitations of this study arise from the retrospective nature of observation of examinations performed for clinical purposes. The ETV studies were not per- 
Table 5: Clinical outcomes at 6 months and last follow-up after ETV ${ }^{\text {a }}$

\begin{tabular}{|c|c|c|c|c|c|c|c|}
\hline & \multicolumn{2}{|c|}{ Improved } & \multicolumn{2}{|c|}{ Same as Baseline } & \multicolumn{2}{|c|}{ Worse Compared with Baseline } & \multirow[b]{2}{*}{$\begin{array}{c}P \\
\text { Value }\end{array}$} \\
\hline & $\begin{array}{c}\text { NICO } \\
\text { Myriad Used }\end{array}$ & $\begin{array}{c}\text { No } \\
\text { NICO Myriad }\end{array}$ & $\begin{array}{c}\text { NICO } \\
\text { Myriad Used }\end{array}$ & $\begin{array}{c}\text { No } \\
\text { NICO Myriad }\end{array}$ & $\begin{array}{c}\text { NICO } \\
\text { Myriad Used }\end{array}$ & $\begin{array}{c}\text { No } \\
\text { NICO Myriad }\end{array}$ & \\
\hline \multicolumn{8}{|c|}{ Headache } \\
\hline $6 \mathrm{Mo}$ & $88.9 \%$ & $80 \%$ & $11.1 \%$ & $13.3 \%$ & $0 \%$ & $6.7 \%$ & $>.05$ \\
\hline LFU & $77.8 \%$ & $53.3 \%$ & $22.2 \%$ & $33.3 \%$ & $0 \%$ & $13.3 \%$ & $>.05$ \\
\hline \multicolumn{8}{|c|}{ Dizziness } \\
\hline $6 \mathrm{Mo}$ & $100 \%$ & $83.3 \%$ & $0 \%$ & $16.7 \%$ & $0 \%$ & $0 \%$ & $>.05$ \\
\hline LFU & $100 \%$ & $83.3 \%$ & $0 \%$ & $16.7 \%$ & $0 \%$ & $0 \%$ & $>.05$ \\
\hline \multicolumn{8}{|l|}{ Nausea } \\
\hline $6 \mathrm{Mo}$ & $75 \%$ & $75 \%$ & $25 \%$ & $25 \%$ & $0 \%$ & $0 \%$ & $>.05$ \\
\hline LFU & $50 \%$ & $75 \%$ & $50 \%$ & $25 \%$ & $0 \%$ & $0 \%$ & $>.05$ \\
\hline \multicolumn{8}{|c|}{ Vision deficit } \\
\hline $6 \mathrm{Mo}$ & $83.3 \%$ & $77.8 \%$ & $16.7 \%$ & $22.2 \%$ & $0 \%$ & $0 \%$ & $>.05$ \\
\hline LFU & $83.3 \%$ & $77.8 \%$ & $16.7 \%$ & $22.2 \%$ & $0 \%$ & $0 \%$ & $>.05$ \\
\hline \multicolumn{8}{|c|}{ Gait abnormality } \\
\hline $6 \mathrm{Mo}$ & $100 \%$ & $100 \%$ & $0 \%$ & $0 \%$ & $0 \%$ & $0 \%$ & $>.05$ \\
\hline LFU & $100 \%$ & $70 \%$ & $0 \%$ & $10 \%$ & $0 \%$ & $20 \%$ & $>.05$ \\
\hline \multicolumn{8}{|c|}{ Urinary incontinence } \\
\hline $6 \mathrm{Mo}$ & $100 \%$ & $100 \%$ & $0 \%$ & $0 \%$ & $0 \%$ & $0 \%$ & $>.05$ \\
\hline LFU & $85.7 \%$ & $85.7 \%$ & $0 \%$ & $0 \%$ & $14.3 \%$ & $14.3 \%$ & $>.05$ \\
\hline \multicolumn{8}{|c|}{ Cognitive dysfunction } \\
\hline $6 \mathrm{Mo}$ & $87.5 \%$ & $70 \%$ & $12.5 \%$ & $30 \%$ & $0 \%$ & $0 \%$ & $>.05$ \\
\hline LFU & $87.5 \%$ & $70 \%$ & $12.5 \%$ & $20 \%$ & $0 \%$ & $10 \%$ & $>.05$ \\
\hline
\end{tabular}

Note:-LFU indicates last follow-up.

a Outcomes are given among those with preoperative symptoms.

formed at regular intervals following the procedure, possibly due to follow-up studies being performed outside the institution or within the institution but not using the high-resolution ETV MR imaging protocol. We have accounted for this issue partly by recording the elapsed time from the ETV operation to each MR imaging study. The time to the first follow-up ranged from a few hours after the operation to almost 2 and a half years after the ETV operation.

Because examinations were performed for clinical purposes, symptomatic patients who eventually had failed ETV may be over-represented in our sample. Although phase-contrast imaging was part of the high-resolution ETV protocol, it was not used in this study. Follow-up MRIs were performed to clinically ensure the patency of the ETV defect and the success of the ventriculostomy, in addition to its research role as a high-resolution imaging technique. This follow-up imaging protocol has been established as a standard at the current institution to aid in the evaluation of ETV patency. To accomplish this study, we included all patients fitting the inclusion criteria and having undergone ETV. ETV has been successful in multiple disease processes, including a subsection of patients with refractory long-standing symptomatic normal pressure hydrocephalus with overt ventriculomegaly and other unknown causes of hydrocephalus. ${ }^{19-23}$ The inclusion of this case represents a rare indication for ETV and did not significantly impact the results of the study.

\section{CONCLUSIONS}

High-resolution MR imaging with sagittal CISS images is useful in the postoperative evaluation of ETVs. A small percentage closed in the first year of follow-up with an increase in size of the ETV defect in all remaining patients, without premature defect closure. Such findings may prove useful in determining the optimal dura- tion of follow-up with MR imaging of patients who have undergone ETV.

Disclosures: Ari Blitz—UNRELATED: Consultancy: Guerbet; Grants/Grants Pending: Aesculab, National Institutes of Health, Comments: FAIN U01DC013778, R21 NS096497*; Support for Travel to Meetings for the Study or Other Purposes: International Society for Hydrocephalus and CSF Disorders, Comments: funding for travel as a keynote speaker; Travel/Accommodations/Meeting Expenses Unrelated to Activities Listed: French-Israeli radiology course, Comments: educational talk, partial travel reimbursement. *Money paid to the institution.

\section{REFERENCES}

1. Di Rocco C, Massimi L, Tamburrini G. Shunts vs endoscopic third ventriculostomy in infants: are there different types and/or rates of complications? A review. Childs Nerv Syst 2006 22:1573-89 Medline

2. Santamarta D, Martin-Vallejo J, Díaz-Alvarez A, et al. Changes in ventricular size after endoscopic third ventriculostomy. Acta Neurochir (Wien) 2008;150:119-27; discussion 127 CrossRef Medline

3. van Beijnum J, Hanlo PW, Fischer K, et al. Laser-assisted endoscopic third ventriculostomy: long-term results in a series of 202 patients. Neurosurgery 2008;62:437-43; discussion 443-44 CrossRef Medline

4. Yadav YR, Parihar V, Pande S, et al. Endoscopic third ventriculostomy. J Neurosci Rural Pract 2012;3:163-73 CrossRef Medline

5. Anık I, Ceylan S, Koc K, et al. Membranous structures affecting the success of endoscopic third ventriculostomy in adult aqueductus sylvii stenosis. Minim Invasive Neurosurg 2011;54:68-74 CrossRef Medline

6. Antes S, Tschan CA, Oertel JM. An operative technique combining endoscopic third ventriculostomy and long-term ICP monitoring. Childs Nerv Syst 2014;30:331-35 CrossRef Medline

7. Dinçer A, Yildiz E, Kohan S, et al. Analysis of endoscopic third ventriculostomy patency by MRI: value of different pulse sequences, the sequence parameters, and the imaging planes for investigation of flow void. Childs Nerv Syst 2011;27:127-35 CrossRef Medline

8. Feng HL, Huang GF, Liao XL, et al. Endoscopic third ventriculostomy in the management of obstructive hydrocephalus: an outcome analysis. J Neurosurg 2004;100:626-33 CrossRef Medline 
9. Mobbs RJ, Vonau M, Davies MA. Death after late failure of an endoscopic third ventriculostomy: a potential solution. Neurosurgery 2003;53:384-85; discussion 385-86 CrossRef Medline

10. Fukuhara T, Luciano MG, Kowalski RJ. Clinical features of third ventriculostomy failures classified by fenestration patency. Surg Neurol 2002;58:102-10 CrossRef Medline

11. Goodwin CR, Sankey EW, Jusue-Torres I, et al. The use of an aspirating/resecting device to reduce stoma closure following endoscopic third ventriculostomy for aqueductal stenosis. Oper Neurosurg (Hagerstown) 2015;11:512-17 CrossRef Medline

12. Bargalló N, Olondo L, Garcia AI, et al. Functional analysis of third ventriculostomy patency by quantification of CSF stroke volume by using cine phase-contrast MR imaging. AJNR Am J Neuroradiol 2005;26:2514-21 Medline

13. Dlouhy BJ, Capuano AW, Madhavan K, et al. Preoperative third ventricular bowing as a predictor of endoscopic third ventriculostomy success. J Neurosurg Pediatr 2012;9:182-90 CrossRef Medline

14. Jallo GI, Kothbauer KF, Abbott IR. Endoscopic third ventriculostomy. Neurosurg Focus 2005;19:E11

15. Fischbein NJ, Ciricillo SF, Barr RM, et al. Endoscopic third ventriculocisternostomy: MR assessment of patency with 2-D cine phase-contrast versus T2-weighted fast spin echo technique. Pediatr Neurosurg 1998;28:70-78 CrossRef Medline

16. Hoffmann KT, Lehmann TN, Baumann C, et al. CSF flow imaging in the management of third ventriculostomy with a reversed fast im- aging with steady-state precession sequence. Eur Radiol 2003;13: 1432-37 Medline

17. Kulkarni AV, Drake JM, Armstrong DC, et al. Imaging correlates of successful endoscopic third ventriculostomy. J Neurosurg 2000;92: 915-19 CrossRef Medline

18. Sankey EW, Elder BD, Liu A, et al. Predictors of admission and shunt revision during emergency department visits for shunt-treated adult patients with idiopathic intracranial hypertension. J Neurosurg 2017;127:233-39 CrossRef Medline

19. Balevi M. Endoscopic third ventriculostomy in normal pressure hydrocephalus and symptomatic long-standing overt ventriculomegaly. Asian J Neurosurg 2017;12:605-12 CrossRef Medline

20. Gangemi M, Maiuri F, Naddeo M, et al. Endoscopic third ventriculostomy in idiopathic normal pressure hydrocephalus: an Italian multicenter study. Neurosurgery 2008;63:62-67; discussion 67-69 Medline

21. Hailong F, Guangfu H, Haibin T, et al. Endoscopic third ventriculostomy in the management of communicating hydrocephalus: a preliminary study. J Neurosurg 2008;109:923-30 CrossRef Medline

22. Kiefer M, Eymann R, Steudel WI. LOVA hydrocephalus: a new entity of chronic hydrocephalus [in German]. Nervenarzt 2002;73:972-81 CrossRef Medline

23. Grand W, Leonardo J, Chamczuk AJ, et al. Endoscopic third ventriculostomy in $\mathbf{2 5 0}$ adults with hydrocephalus: patient selection, outcomes, and complications. Neurosurgery 2016;78:109-19 